\title{
Unknown Disturbance Estimation for a PMSM with a Hybrid Sliding Mode Observer
}

\author{
Gang Chen, ${ }^{1,2}$ Yong Zhou, ${ }^{3}$ TingTing Gao, ${ }^{2}$ and Qicai Zhou ${ }^{1}$ \\ ${ }^{1}$ Tongii University, Shanghai 200092, China \\ ${ }^{2}$ Zhejiang Textile \& Fashion College, Ningbo 315211, China \\ ${ }^{3}$ Wild SC Intelligent Technology CO., LTD, Ningbo 315500, China
}

Correspondence should be addressed to Yong Zhou; yong.zhou@wildsc.com.cn

Received 7 July 2014; Revised 26 September 2014; Accepted 2 October 2014

Academic Editor: Xudong Zhao

Copyright (C) 2015 Gang Chen et al. This is an open access article distributed under the Creative Commons Attribution License, which permits unrestricted use, distribution, and reproduction in any medium, provided the original work is properly cited.

\begin{abstract}
A hybrid sliding mode observer that combines high gain feedback and a high-order sliding mode term is developed to identify the time-varying disturbance for a permanent-magnet synchronous motor (PMSM). Based on the measurable current and the position, the unknown disturbance can be identified from the sliding mode term without digital filter effect. It is then used to enhance the robustness of the speed control dynamics. For ease of implementation in real applications, such as DSP and FPGA, the proposed observer is properly designed to avoid complex mathematical operators. Simulation results are given to illustrate the performance of the proposed observer.
\end{abstract}

\section{Introduction}

In real industrial application, the unknown disturbance on a permanent-magnet synchronous motor (PMSM) is inevitable and it limits the performance of the controlled processes. For such a situation, a robust observer with high estimation accuracy is required to recover the uncertainty in real time.

The sliding-mode-based observer has been proven to be an effective approach for handling uncertain systems, due to its capability of reconstructing the uncertainties based on the equivalent injection input concept [1-3], and there are many effective results presented during past years [413]. In [14], a sliding mode flux observer is developed for an induction motor, and the unknown rotor resistance can be reconstructed from a switching function after the sliding surface driven to zero. In $[15,16]$, the sliding mode observer is used to identify the back-EMFs for a PMSM. However, such results require a low-pass filter for the uncertainties reconstruction because of the discontinuous switching feedback, and the uncertainty estimation accuracy is greatly dependent on the low-pass filter parameters.
To improve the estimation accuracy by removing the filtering effect, some existing works have suggested the usage of a saturation function to replace the switching term [17-19]. However, this results in a trade-off between the robustness of observer and the estimation accuracy of uncertainty. Another existing method is based on the higher-order sliding mode techniques, by treating the derivatives of the system input as a new control signal, which results in a continuous integral function of the switching term; then the chattering/filtering effect can be avoided [20,21].

Another problem is that the sliding mode gains have to be chosen large enough to ensure the stability of the observer, which is usually related to the system initial values. To solve this problem, a hybrid observer that combines high gain feedback and higher-order sliding mode observer has been proposed in [22], in which the high gain feedback works to constrain the estimation error within an invariant set regardless of initial values; then the sliding mode gains can be designed to ensure the global stability of the observer. Such hybrid observers have been applied into a series DC motor for the speed estimation and unknown time-varying parameter identification for a DC motor [23]. 
In this paper, we will consider the unknown disturbance estimation for a permanent-magnet synchronous motor. A hybrid sliding mode observer is developed for the disturbance identification. The most challenging problem is how to properly design the observer algorithm to simplify the observer implementation in real applications, such as in a digital signal processor (DSP) and FPGA. The contribution of this work can be summarized as follows.

(1) With the proposed observer, the disturbance $T_{L}$ can be asymptotically estimated and then used to enhance the robustness of the speed control dynamics. The theoretical analysis and simulation results are given to demonstrate the effectiveness of the proposed observer.

(2) A hybrid observer structure is adopted, in which the high gain feedback term works to speed up the convergence time and guarantees the sliding mode gain design is independent of the system initial values.

(3) For disturbance identification, the digital filtering effect is successfully avoided without sacrificing robustness of the observer.

(4) The twisting sliding mode algorithm is properly integrated in the proposed observer, to make the observer ease for implementation in real application.

The remainder of this paper is organized as follows. Section 2 introduces the mathematical mode of the PMSM and some background results are presented. In Section 3, a speed observer and a disturbance are developed to identify the unknown external load disturbance. In Section 4, some simulations are provided to illuminate the effectiveness of the two proposed observers. Section 5 concludes this paper.

\section{Preliminary}

2.1. Mathematical Model. The state equation of a surfacemounted magnet brushless AC motor in stationary $\alpha-\beta$ reference frame is given by [24]:

$$
\begin{aligned}
& \dot{i}_{\alpha}=-\frac{R}{L} i_{\alpha}+\frac{\lambda_{m}}{L} \omega \sin \theta+\frac{u_{\alpha}}{L}, \\
& \dot{i}_{\beta}=-\frac{R}{L} i_{\beta}-\frac{\lambda_{m}}{L} \omega \cos \theta+\frac{u_{\beta}}{L}, \\
& \dot{\theta}=\omega \\
& \dot{\omega}=\frac{3}{2} \frac{p^{2}}{J} \lambda_{m}\left(-i_{\alpha} \sin \theta+i_{\beta} \cos \theta\right)-\frac{B}{J} \omega-p \frac{T_{L}}{J},
\end{aligned}
$$

where $i_{\alpha, \beta}$ is the state current in $\alpha$ - $\beta$ reference frame, $u_{\alpha, \beta}$ is the state voltage in $\alpha$ - $\beta$ reference frame, $R$ is the resistance, $L$ is the inductance, $\lambda_{m}$ is the flux-linkage due to permanent magnet, $\theta$ is the rotor position, $\omega$ is the electrical angular speed, $J$ is the rotor moment of inertia, $B$ is the viscousfriction coefficient, and $T_{L}$ is the load torque.

Here, the load torque $T_{L}$ is assumed to be the system uncertainty that includes the unknown external disturbance, the parameter variations of rotor inertia, and the viscousfriction coefficient.

The target of this paper is to develop a robust and high accuracy observer that can identify the unknown disturbance $T_{L}$, based on the measurable current, voltage, and rotor position.

2.2. Background Results. Consider an uncertain nonlinear system in the form of

$$
\begin{aligned}
& \dot{\mathbf{x}}=\mathbf{A} \mathbf{x}+\alpha(\mathbf{x}, u)+\mathbf{P}(\mathbf{x}) d(t), \\
& y=\mathbf{C} \mathbf{x},
\end{aligned}
$$

where $\mathbf{x}=\left[x_{1}, x_{2}, \ldots, x_{n}\right]^{T} \in \mathfrak{R}^{n}$ and

$$
\begin{aligned}
& \mathbf{A}=\left[\begin{array}{cc}
\mathbf{0}_{(n-1) \times 1} & \mathbf{I}_{(n-1) \times(n-1)} \\
\mathbf{0}_{1 \times 1} & \mathbf{0}_{1 \times(n-1)}
\end{array}\right], \\
& \mathbf{C}=\left[\begin{array}{llll}
1 & 0 & \cdots & 0
\end{array}\right] \in \mathfrak{R}^{n}
\end{aligned}
$$

are constant matrices; $d(t) \in \mathfrak{R}$ denotes the lumped system uncertainty; $u$ is the system input; the nonlinear functions $\alpha(\mathbf{x}, u)$ and $\mathbf{P}(\mathbf{x})$ are smooth Lipschitz vectors with triangular structures, it has

$$
\begin{aligned}
& \alpha(\mathbf{x})=\left[0, \ldots, 0, \alpha_{r}\left(x_{1}, \ldots, x_{r}\right), \ldots, \alpha_{n}\left(x_{1}, \ldots, x_{n}\right)\right]^{T} \\
& \mathbf{P}(\mathbf{x})=\left[0, \ldots, 0,1, p_{r+1}\left(x_{1}, \ldots, x_{r}\right), \ldots, p_{n}\left(x_{1}, \ldots, x_{n}\right)\right]^{T}
\end{aligned}
$$

with $r$ being the relative degree order between the disturbance $d(t)$ and the measurable output $y$.

Then, a hybrid observer that combines high gain feedback with higher-order sliding mode can be designed as [22]

$$
\begin{aligned}
& \dot{\hat{\mathbf{x}}}=\mathbf{A} \widehat{\mathbf{x}}+\alpha(\widehat{\mathbf{x}}, u)+\mathbf{L}(y-\mathbf{C} \widehat{\mathbf{x}})+\mathbf{P}(\widehat{\mathbf{x}}) k_{r} u_{r}, \\
& \dot{u}_{r}= \begin{cases}0, & \text { if } u_{r} \geq \bar{u}_{r}, \\
v, & \text { if } u_{r}<\bar{u}_{r},\end{cases}
\end{aligned}
$$

where $\mathbf{L}$ is the high gain linear feedback parameter, given by

$$
\mathbf{L}=\left[\begin{array}{llll}
l_{1} & l_{2} & \cdots & l_{n}
\end{array}\right]^{T} \in \mathfrak{R}^{n},
$$

and $k_{r}$ is a positive tuning parameter of nonlinear feedback $u_{r}$, which is designed as an integral function of a $(r+1)$ thorder sliding mode term $v ; \bar{u}_{r}$ denotes the upper bound of $u_{r}$.

It has been proven in [22] that the high gain feedback term works to constrain the estimation error to within an invariant set and the nonlinear term $k_{r} u_{r}$ will asymptotically track the unknown disturbance $d(t)$ without digital filtering effect if we properly design the sliding mode feedback term.

The mechanical dynamics given in (2) can be considered as a second-order nonlinear system in (3), with the relative degree order between the disturbance and the measurable output being two; that is, $r=n=2, d(t)=-p T_{L} / J$. Then, one can design a hybrid observer in the form of (6), with $v$ being third-order sliding mode feedback. However, to the best of our understanding, the existing third-order 
or higher-order sliding mode algorithms involve complex math operations that require more computation resources in real implementation, such as in digital signal processor and FPGA.

In next section, we will design a hybrid observer that is based on the second-order twisting sliding mode algorithm [1]; the most advantage of such sliding algorithm is that it only requires the sign of the first derivative operator, but not its real value. Thus, the proposed observer takes less computation resources and is easy for implementation in digital signal processor.

\section{Observer Design}

Consider the mechanical dynamic system described in (2); the rotor position $\theta$ is measurable, and the target is to estimate the unknown disturbance $T_{L}$, as well as the angular speed $\omega$.

It is well-known that there are many methodologies to estimate the angular speed $\omega$ based on the measurable position $\theta$. One common method is based on the backforward differentiator; that is, $\widehat{\omega}_{k}=\left[\theta_{k}-\theta_{(k-1)}\right] / T_{s}$, where $T_{s}$ is the sampling time and subscript $k$ denotes the variable value at time $t=k T_{s}$. For such method, the estimation error is proportional to sampling time. On the other hand, small sampling time may enlarge the numerical error of $\left(\theta_{k}-\right.$ $\left.\theta_{k-1}\right)$, especially at low rotor speed and low position sensor resolution situation.

As mentioned in the previous section, in order to simplify the hybrid observer structure and remove the digital filtering effect, we will consider using the second-order twisting algorithm for the unknown disturbance estimation.

The system diagram is shown in Figure 1. The first observer (speed observer) will be performed to identify the rotor speed without involving the operation of inverse of sampling time, that is, $1 / T_{s}$. Then the second observer (disturbance observer) ensures the unknown disturbance $T_{L}$ can be identified online without digital filtering effect. Finally, one can use the identified disturbance to compensate the control loop to improve the robustness of the control system.

3.1. Speed Observer. By considering the rotor speed $\omega$ as the system uncertainty, a hybrid sliding mode observer is designed in the form of

$$
\dot{\hat{\theta}}=-l_{1} e_{1}+u_{1}
$$

where $e_{1} \triangleq \widehat{\theta}-\theta, l_{1}$ is a positive high gain parameter, and $u_{1}$ is the nonlinear feedback which is given by

$$
\begin{aligned}
& \dot{u}_{1}= \begin{cases}0, & \text { if }\left|u_{1}\right| \geq \bar{u}_{1}, \\
v_{1}, & \text { if }\left|u_{1}\right|<\bar{u}_{1},\end{cases} \\
& v_{1}=-\rho_{11} \operatorname{sign}(\widehat{\theta}-\theta)-\rho_{12} \operatorname{sign}(\dot{\hat{\theta}}-\dot{\theta}) .
\end{aligned}
$$

Here, $\bar{u}_{1}$ is the upper bound of the nonlinear feedback term, and it is chosen large enough, as $\bar{u}_{1} \geq|\omega|$. Then, $\rho_{11}$ and $\rho_{12}$ are the properly chosen positive sliding gains.

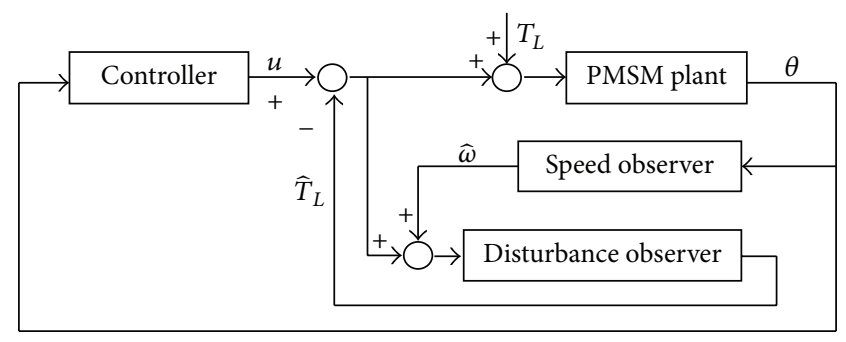

FIGURE 1: System diagram.

Theorem 1. With the proposed hybrid observer given in (8) and (9), the rotor speed can be asymptotically identified by properly choosing the observer parameters; that is, it will have $\omega=u_{1}$.

Proof. Compare the equations in (2) and (8); the estimation error dynamic of $e_{1}$ can be written as

$$
\dot{e}_{1}=-l_{1} e_{1}-\omega+u_{1} .
$$

By defining a Lyapunov function $V_{1}=\left|e_{1}\right|^{2} / 2$, it has

$$
\begin{aligned}
\dot{V}_{1} & =-l_{1}\left|e_{1}\right|^{2}-\omega e_{1}+u_{1} e_{1} \\
& \leq\left|e_{1}\right|\left(-l_{1}\left|e_{1}\right|+|\omega|+\bar{u}_{1}\right) \\
& \leq\left|e_{1}\right|\left(-l_{1}\left|e_{1}\right|+2 \bar{u}_{1}\right) \quad\left(\text { choosing } \bar{u}_{1} \geq|\omega|\right)
\end{aligned}
$$

which implies that, after some time, the estimation error $e_{1}$ will converge into an invariant set regardless of initial value of $\theta$; that is, $\left|e_{1}\right| \leq 2 \bar{u}_{1} / l_{1}$.

Now, assuming $u_{1}<\bar{u}_{1}$, we consider the new dynamic by taking the derivative on both sides of (10); it has

$$
\begin{aligned}
\ddot{e}_{1} & =-l_{1} \dot{e}_{1}-\dot{\omega}+v_{1} \\
& =l_{1}^{2} e_{1}+l_{1} \omega-l_{1} u_{1}-\dot{\omega}+v_{1} \\
& \triangleq d_{1}(t)+v_{1}
\end{aligned}
$$

with $d_{1}(t)$ denoting dynamic terms $\left(l_{1}^{2} e_{1}+l_{1} \omega-l_{1} u_{1}-\dot{\omega}\right)$, which is assumed to be bounded with $\bar{d}_{1}$. Then, by defining the second Lyapunov function $V_{2}=\left|\dot{e}_{1}\right|^{2} / 2+\rho_{11}\left|e_{1}\right|$, which is continuously differentiable except on $e_{1}=0$, it can be verified that

$$
\begin{aligned}
\dot{V}_{2}= & \dot{e}_{1} \ddot{e}_{1}+\rho_{11} \dot{e}_{1} \operatorname{sign}\left(e_{1}\right) \\
= & \dot{e}_{1}\left[d_{1}(t)-\rho_{11} \operatorname{sign}\left(e_{1}\right)-\rho_{12} \operatorname{sign}\left(\dot{e}_{1}\right)\right] \\
& +\rho_{11} \dot{e}_{1} \operatorname{sign}\left(e_{1}\right) \\
= & \dot{e}_{1}\left[d_{1}(t)-\rho_{12} \operatorname{sign}\left(\dot{e}_{1}\right)\right] \\
\leq & \left|\dot{e}_{1}\right|\left(\bar{d}_{1}-\rho_{12}\right)
\end{aligned}
$$

which implies that it has $\dot{V}_{2}<0$ if the sliding gains are chosen large enough; that is, $\rho_{11}>\rho_{12}>\bar{d}_{1}$. In other words, 
the second-order sliding surface $\dot{e}_{1}=e_{1}=0$ will be reached in a finite time and remained thereafter. Then, the rotor speed can be directly obtained from (9), as $\omega=u_{1}$.

Remark 2. In real implementation, the derivatives in (9) can be replaced with their backward differentiators, respectively; that is, $v_{1 k}=-\rho_{11} \operatorname{sign}\left(e_{1 k}\right)-\rho_{12} \operatorname{sign}\left(e_{1 k}-e_{1(k-1)}\right)$. The subscript $k$ denotes the variables obtained at sampling time $t=k T_{s}$. Note that the operation of $1 / T_{s}$ is completely avoided; then one can increase the sampling and control frequency without sacrificing its related numerical accuracy.

3.2. Disturbance Observer. With the identified rotor speed, a hybrid disturbance observer is designed in the form of

$$
\begin{aligned}
& \dot{\hat{\omega}}=\frac{3}{2} \frac{p^{2}}{J} \lambda_{m}\left(-i_{\alpha} \sin \theta+i_{\beta} \cos \theta\right)-\frac{B}{J} \widehat{\omega}-l_{2} e_{2}-p \frac{u_{2}}{J}, \\
& \dot{u}_{2}= \begin{cases}0, & \text { if }\left|u_{2}\right| \geq \bar{u}_{2}, \\
v_{2}, & \text { if }\left|u_{2}\right|<\bar{u}_{2},\end{cases} \\
& v_{2}=-\rho_{21} \operatorname{sign}\left(\widehat{\omega}-u_{1}\right)-\rho_{22} \operatorname{sign}\left(\dot{\hat{\omega}}-\dot{u}_{1}\right),
\end{aligned}
$$

where $e_{2} \triangleq \widehat{\omega}-u_{1} ; l_{2}$ is a positive high gain parameter; $\rho_{21}$ and $\rho_{22}$ are two properly chosen positive sliding gains; $\bar{u}_{2}$ is the upper bound of the nonlinear feedback $u_{2}$, which is designed as an integral function of a sliding mode term $v_{2}$.

Theorem 3. With the proposed speed observer in (8) and the disturbance observer in (14), the system unknown disturbance $T_{L}$ can be identified in finite time if properly choosing the observer parameters; that is, it will have $T_{L}=u_{2}$.

Proof. According to the result in Theorem 1, the rotor speed $\omega$ can be identified from $u_{1}$ after some time; therefore, the dynamics of the estimation error $e_{2}=\widehat{\omega}-u_{1}=\widehat{\omega}-\omega$ can be deduced from (2) and (14) as

$$
\dot{e}_{2}=-\frac{B}{J} e_{2}-l_{2} e_{2}+p \frac{T_{L}}{J}-p \frac{u_{2}}{J} .
$$

Noting that the dynamic form in (15) is similar to the one in (10), then it can be proven that the second-order sliding surface $\dot{e}_{2}=e_{2}=0$ will be reached and remained thereafter in finite time, if properly choosing the observer parameters; that is, $\bar{u}_{2}>\left|T_{L}\right| ; \rho_{21}$ and $\rho_{22}$ are chosen large enough.

By substituting the sliding surface $\dot{e}_{2}=e_{2}=0$ into the estimation error dynamics in (15), it has

$$
T_{L}=u_{2}
$$

TABle 1: Parameters of a PMSM.

\begin{tabular}{lcc}
\hline Pole pairs & $p$ & 4 \\
Rating speed & $\omega$ & $600 \mathrm{rad} / \mathrm{s}$ \\
Resistance & $R$ & $2.5 \Omega$ \\
Inductance & $L$ & $8 \mathrm{mH}$ \\
Inertia & $J$ & $0.00027 \mathrm{~kg} \cdot \mathrm{m}^{2}$ \\
Viscous-friction coefficient & $B$ & $0.04 \mathrm{~N} \cdot \mathrm{m} / \mathrm{rad} / \mathrm{s}$ \\
Field flux-related coefficient & $\lambda_{m}$ & $0.041 \mathrm{~N} \cdot \mathrm{m} / \mathrm{Wb} \cdot \mathrm{A}$ \\
\hline
\end{tabular}
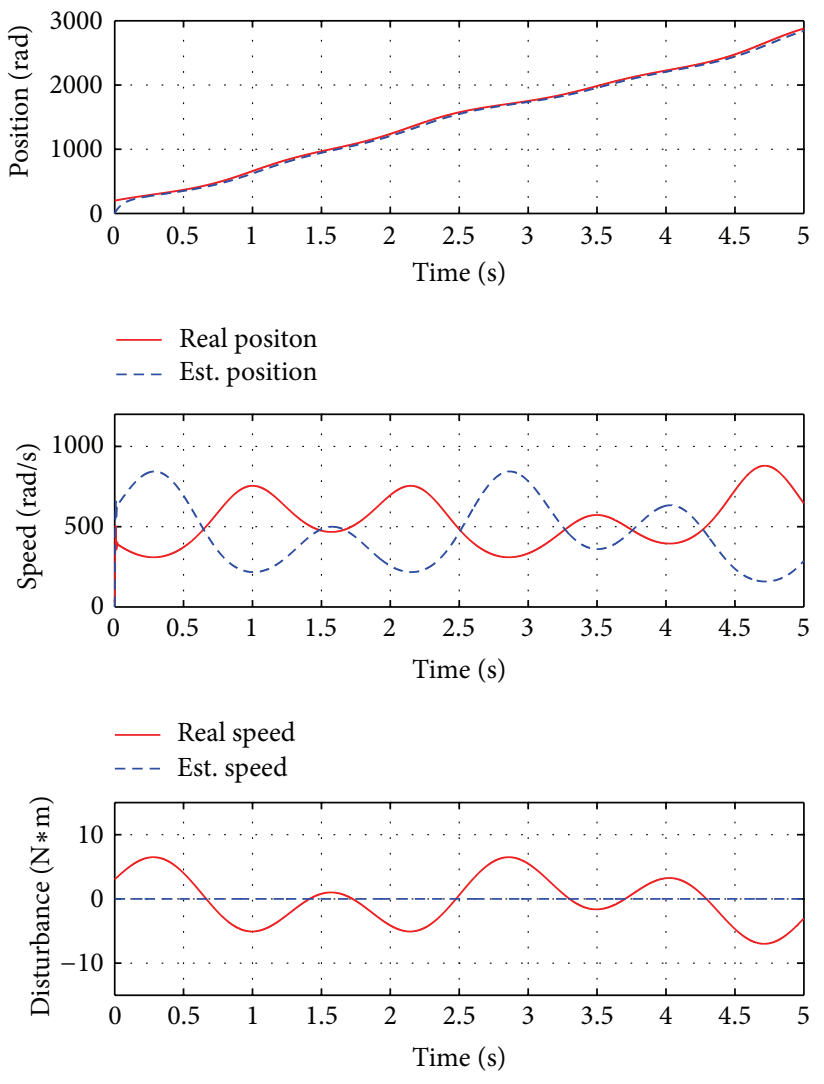

_ Real disturbance
_- - Est. disturbance

Figure 2: Traditional high gain observer.

which means the unknown disturbance can be directly obtained from $u_{2}$ without digital filtering effect.

Remark 4. In real implementation, the derivatives in (14) can be replaced with their backward differentiators, respectively; that is, $v_{2 k}=-\rho_{21} \operatorname{sign}\left(e_{2 k}\right)-\rho_{22} \operatorname{sign}\left(e_{2 k}-e_{2(k-1)}\right)$. Then, the operation of $1 / T_{s}$ is completely avoided.

\section{Simulation Result}

In this section, we consider the simulation on a PMSM with its parameters being given in Table 1 .

To demonstrate the proposed observers performance, a speed open loop control platform is used, with the unknown 

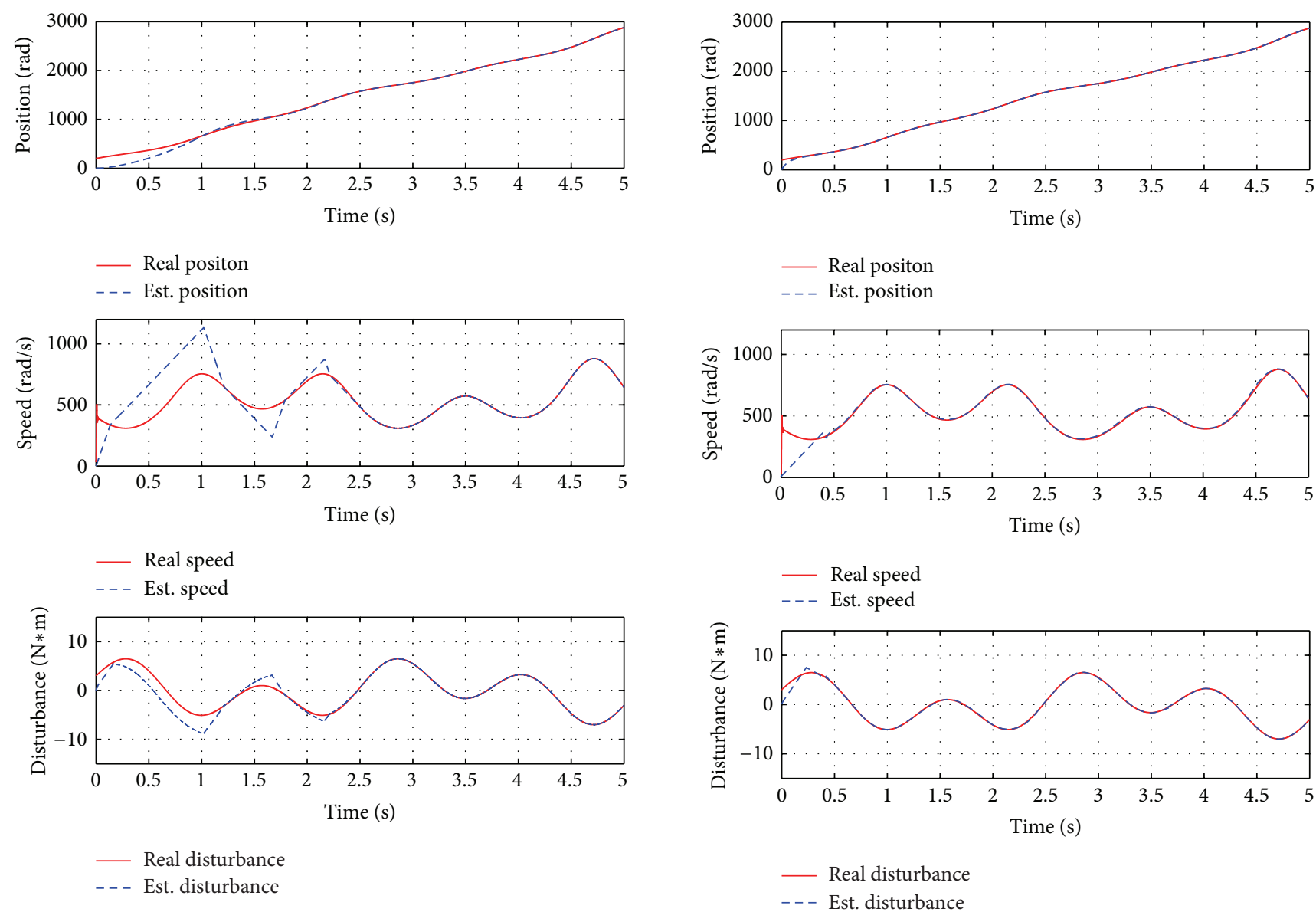

- Real disturbance

- - - Est. disturbance

Figure 3: Pure sliding mode observer.

torque disturbance given as $T_{L}=4 \sin (5 t)+3 \cos (2 t) \mathrm{N} \cdot \mathrm{m}$. Then, the estimation performance among a traditional high gain observer, a pure higher-order sliding mode observer, and the proposed hybrid observer is carefully compared.

Furthermore, the speed closed loop control performance with and without the compensation from identified disturbance is also addressed.

4.1. Traditional High Gain Observer. First, a traditional high gain observer is applied by setting the sliding mode feedback terms in (9) and (14) to zeros; that is, $u_{1}=0, u_{2}=0$, and $e_{2} \triangleq \widehat{\omega}-\omega$. And the high gain parameters are chosen as $l_{1}=20$ and $l_{2}=20$. The initial rotor position and speed are set to $\omega(0)=50 \mathrm{rad} / \mathrm{s}$ and $\theta(0)=200 \mathrm{rad}$. Then, the simulation result is shown in Figure 2.

It can be seen that the high gain observer failed to estimate the unknown disturbance as it does not have any mechanism to identify it.

4.2. Pure Sliding Mode Observer. Then, a pure sliding mode observer is used to identify the unknown disturbance by setting the high gains in (8) and (14) to zeros; that is, $l_{1}=0$ and $l_{2}=0$. Then, the sliding mode parameters are chosen as $k_{11}=1700, k_{12}=800, k_{21}=51$, and $k_{22}=20$. The initial conditions are chosen the same as above.
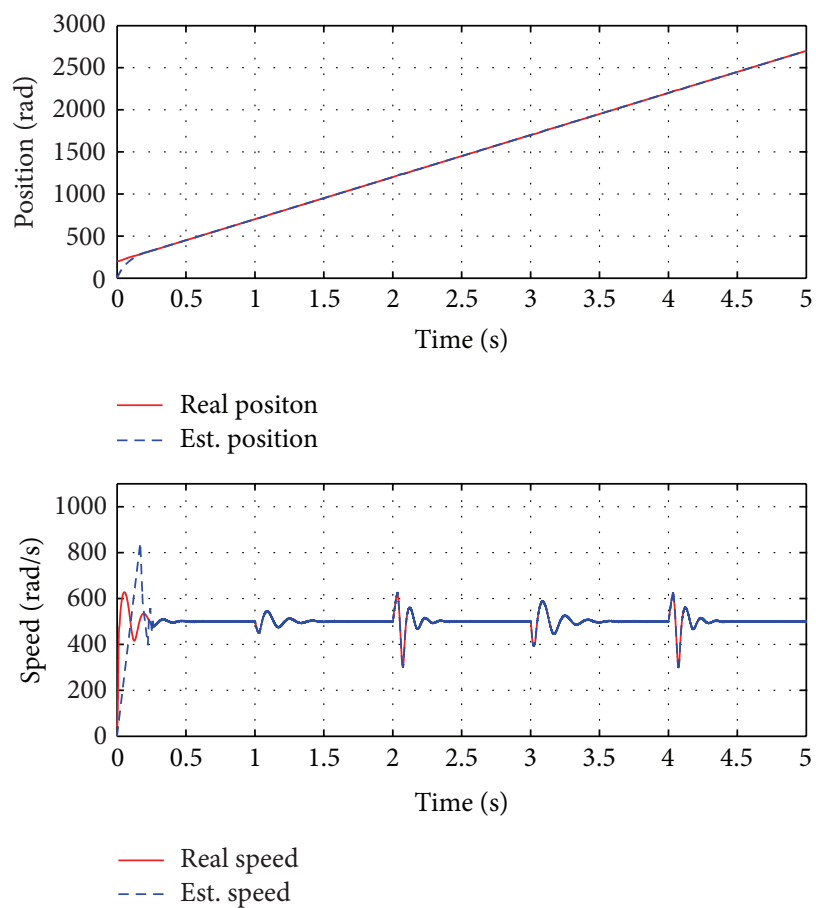

FIGURE 5: Closed loop control without disturbance compensation 1. 


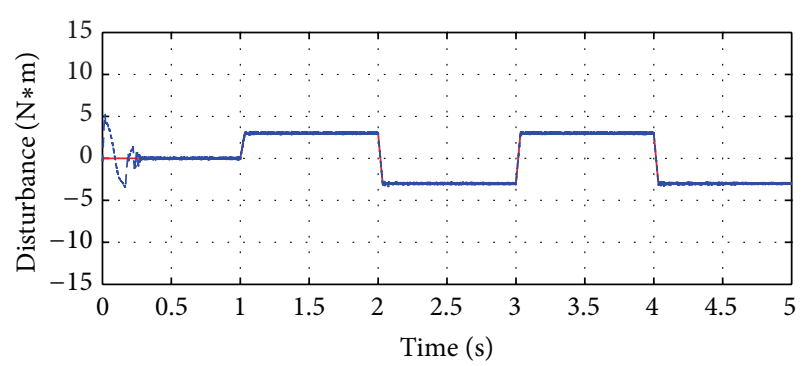

_ Real disturbance

- - - Est. disturbance

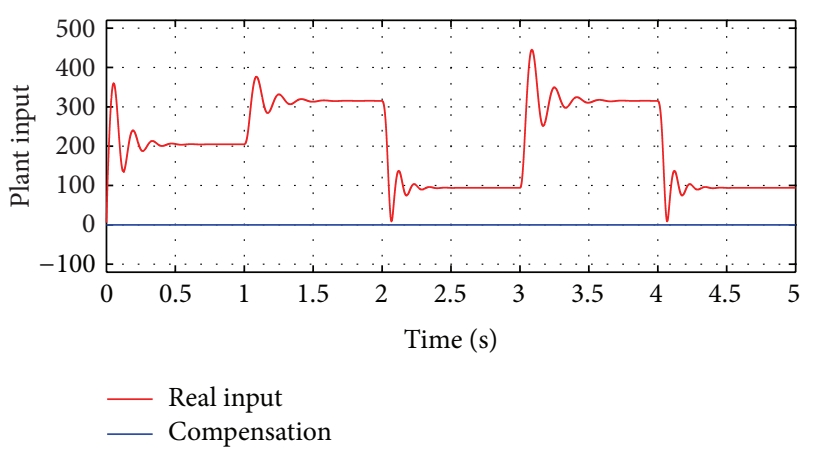

FIGURE 6: Closed loop control without disturbance compensation 2.

The simulation result is shown in Figure 3. Here, we can see that the unknown disturbance can be successfully identified after around 2.4 seconds when the sliding mode surfaces are reached.

4.3. Proposed Hybrid Sliding Mode Observer. Last, the proposed hybrid sliding mode observers in (8)-(14) are applied. The parameters and initial conditions are chosen the same as above, and the simulation result is shown in Figure 4.

It is clear that, with the proposed hybrid sliding mode observer, the unknown disturbance $T_{L}$ can be identified with high accuracy and much faster than the pure sliding mode observer because of the additional high gain feedback terms.

\subsection{Closed Loop Control without Disturbance Compensation.} Now, we consider the speed closed loop control system with unknown disturbance and the system diagram is given in Figure 1.

First, by removing the disturbance compensation from the speed feedback loop, that is, setting $\widehat{T}_{L}=0$, the simulation of speed closed loop control system under a periodic and trapezoidal unknown disturbance is given in Figures 5 and 6.

It can be seen that $300 \mathrm{rad} / \mathrm{sec}$ maximin speed transient is observed when the disturbance changes from $+4 \mathrm{~N} \cdot \mathrm{m}$ to $-4 \mathrm{~N} \cdot \mathrm{m}$ at time of 2 seconds.

4.5. Closed Loop Control with Disturbance Compensation. Then, by setting the disturbance compensation in the speed feedback control loop, that is, $\widehat{T}_{L}=u_{2}$, all the controller and observer parameters are chosen the same as previous.
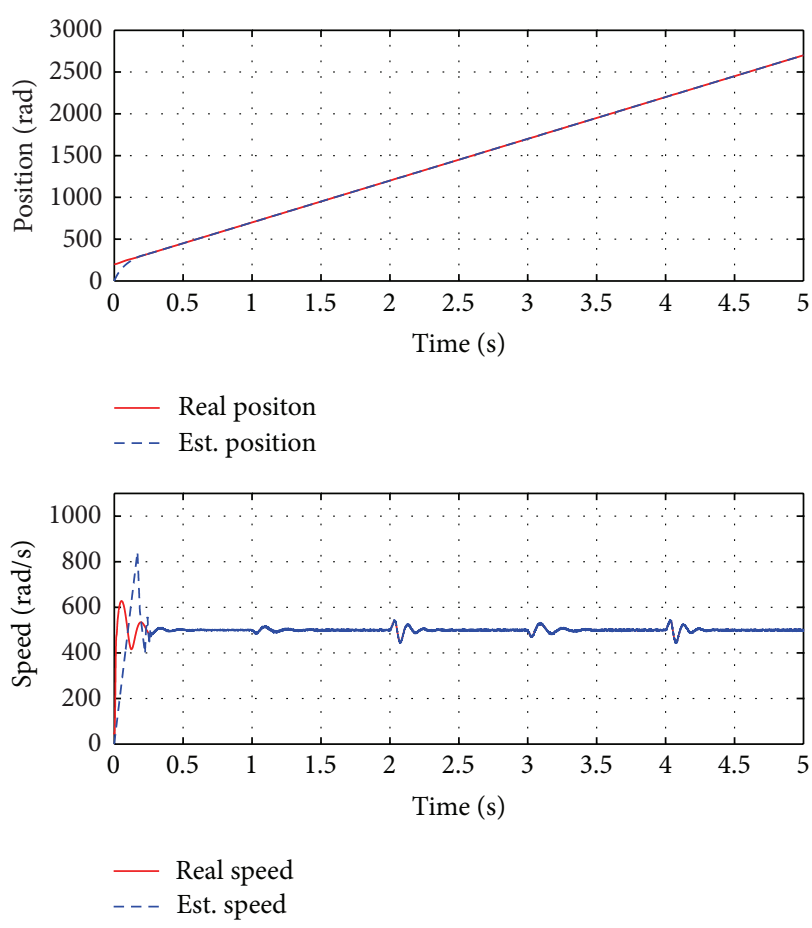

FIGURE 7: Closed loop control with disturbance compensation 1.

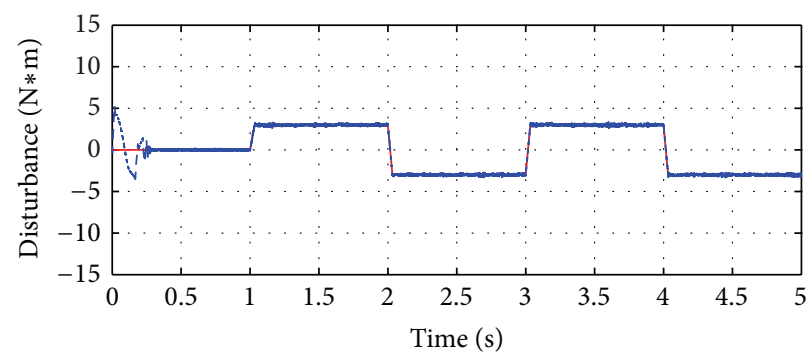

_ Real disturbance

- - - Est. disturbance

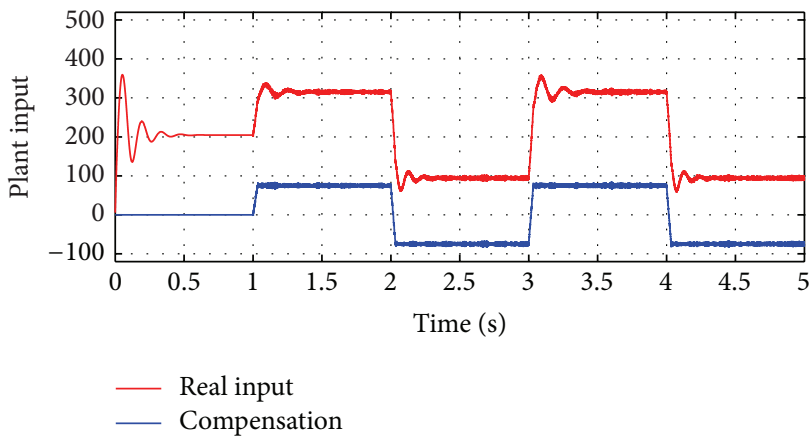

FIGURE 8: Closed loop control with disturbance compensation 2.

The simulation results are shown in Figures 7 and 8 and around $100 \mathrm{rad} / \mathrm{sec}$ speed transient is observed when the disturbance changes from $+4 \mathrm{~N} \cdot \mathrm{m}$ to $-4 \mathrm{~N} \cdot \mathrm{m}$, which means the robustness of the speed closed loop controller is improved with the disturbance compensation. 


\section{Conclusion}

Based on the measurable current and rotor position, a hybrid sliding mode observer is developed for the unknown disturbance estimation on a permanent-magnetic AC motor. The proposed observer has a simple structure and ease for real implementation in digital signal processor, and the unknown disturbance will be identified without digital filtering effect. Moreover, the identified disturbance can be used to compensate online the speed closed loop control system to improve the robustness.

\section{Conflict of Interests}

The authors declare that there is no conflict of interests regarding the publication of this paper.

\section{References}

[1] A. Levant, "Sliding order and sliding accuracy in sliding mode control," International Journal of Control, vol. 58, no. 6, pp. 12471263, 1993.

[2] A. Levant, "Higher-order sliding modes, differentiation and output-feedback control," International Journal of Control, vol. 76, no. 9-10, pp. 924-941, 2003.

[3] V. Utkin, J. Guldner, and J. X. Shi, Sliding Mode Control in Electro-Mechanical Systems, Taylor \& Francis, London, UK, 2nd edition, 2009.

[4] S. K. Spurgeon, "Sliding mode observers: a survey," International Journal of Systems Science: Principles and Applications of Systems and Integration, vol. 39, no. 8, pp. 751-764, 2008.

[5] C. Lascu, I. Boldea, and F. Blaabjerg, "A class of speed-sensorless sliding-mode observers for high-performance induction motor drives," IEEE Transactions on Industrial Electronics, vol. 56, no. 9, pp. 3394-3403, 2009.

[6] M. Ghanes and G. Zheng, "On sensorless induction motor drives: sliding-mode observer and output feedback controller," IEEE Transactions on Industrial Electronics, vol. 56, no. 9, pp. 3404-3413, 2009.

[7] G. H. B. Foo and M. F. Rahman, "Direct torque control of an IPM-synchronous motor drive at very low speed using a sliding-mode stator flux observer," IEEE Transactions on Power Electronics, vol. 25, no. 4, pp. 933-942, 2010.

[8] G. Bartolini, A. Damiano, G. Gatto, I. Marongiu, A. Pisano, and E. Usai, "Robust speed and torque estimation in electrical drives by second-order sliding modes," IEEE Transactions on Control Systems Technology, vol. 11, no. 1, pp. 84-90, 2003.

[9] A. Pisano, A. Davila, L. Fridman, and E. Usai, "Cascade control of PM DC drives via second-order sliding-mode technique," IEEE Transactions on Industrial Electronics, vol. 55, no. 11, pp. 3846-3854, 2008.

[10] M. Iqbal, A. I. Bhatti, S. I. Ayubi, and Q. Khan, "Robust parameter estimation of nonlinear systems using sliding-mode differentiator observer," IEEE Transactions on Industrial Electronics, vol. 58, no. 2, pp. 680-689, 2011.

[11] T. Boileau, N. Leboeuf, B. N. Mobarakeh, and F. M. Tabar, "Online identification of PMSM parameters: parameter identifiability and estimator comparative study," IEEE Transactions on Industrial Electronics, vol. 47, no. 4, pp. 1944-1957, 2011.

[12] M. L. Corradini, G. Ippoliti, S. Longhi, and G. Orlando, "A quasi-sliding mode approach for robust control and speed estimation of PM synchronous motors," IEEE Transactions on Industrial Electronics, vol. 59, no. 2, pp. 1096-1104, 2012.

[13] J. Yang, S. Li, and X. Yu, "Sliding-mode control for systems with mismatched uncertainties via a disturbance observer," IEEE Transactions on Industrial Electronics, vol. 60, no. 1, pp. 160-169, 2013.

[14] A. B. Proca and A. Keyhani, "Sliding-mode flux observer with online rotor parameter estimation for induction motors," IEEE Transactions on Industrial Electronics, vol. 54, no. 2, pp. 716-723, 2007.

[15] S. Chi, Z. Zhang, and L. Y. Xu, "Sliding-mode sensorless control of direct-drive PM synchronous motors for washing machine applications," IEEE Transactions on Industry Applications, vol. 45, no. 2, pp. 582-590, 2009.

[16] V. Delli Colli, R. Di Stefano, and F. Marignetti, "A system-onchip sensorless control for a permanent-magnet synchronous motor," IEEE Transactions on Industrial Electronics, vol. 57, no. 11, pp. 3822-3829, 2010.

[17] G. Foo and M. F. Rahman, "Sensorless sliding-mode MTPA control of an IPM synchronous motor drive using a slidingmode observer and HF signal injection," IEEE Transactions on Industrial Electronics, vol. 57, no. 4, pp. 1270-1278, 2010.

[18] H. Kim, J. Son, and J. Lee, "A high-speed sliding-mode observer for the sensorless speed control of a PMSM," IEEE Transactions on Industrial Electronics, vol. 58, no. 9, pp. 4069-4077, 2011.

[19] Z. Qiao, T. Shi, Y. Wang, Y. Yan, C. Xia, and X. He, "New slidingmode observer for position sensorless control of permanentmagnet synchronous motor," IEEE Transactions on Industrial Electronics, vol. 60, no. 2, pp. 710-719, 2013.

[20] G. Bartolini, A. Ferrara, and E. Usai, "Chattering avoidance by second-order sliding mode control," IEEE Transactions on Automatic Control, vol. 43, no. 2, pp. 241-246, 1998.

[21] Y. Feng, J. Zheng, X. Yu, and N. V. Truong, "Hybrid terminal sliding-mode observer design method for a permanent-magnet synchronous motor control system," IEEE Transactions on Industrial Electronics, vol. 56, no. 9, pp. 3424-3431, 2009.

[22] Y. Zhou, Y. C. Soh, and J. X. Shen, "High-gain observer with higher order sliding mode for state and unknown disturbance estimations," International Journal of Robust and Nonlinear Control, vol. 24, no. 15, pp. 2136-2151, 2013.

[23] Y. Zhou, Y. C. Soh, and J. X. Shen, "Speed estimation and nonmatched time-varying parameter identification for a DC motor with hybrid sliding-mode observer," IEEE Transactions on Industrial Electronics, vol. 60, no. 12, pp. 5539-5549, 2013.

[24] Z. Q. Zhu, X. Zhu, P. D. Sun, and D. Howe, "Estimation of winding resistance and PM flux-linkage in brushless AC machines by reduced-order extended kalman filter," in Proceedings of the IEEE International Conference on Networking, Sensing and Control (ICNSC '07), pp. 740-745, London, UK, April 2007. 


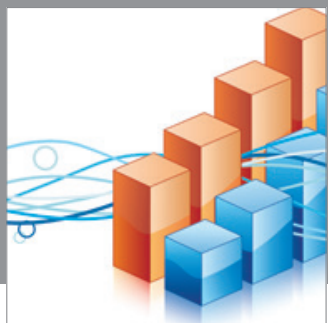

Advances in

Operations Research

mansans

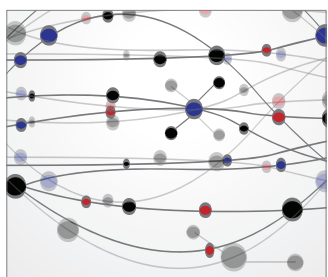

The Scientific World Journal
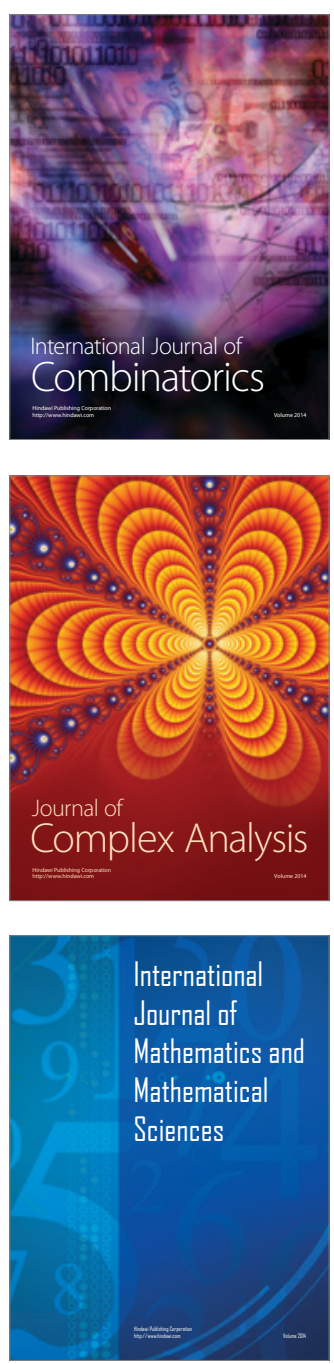
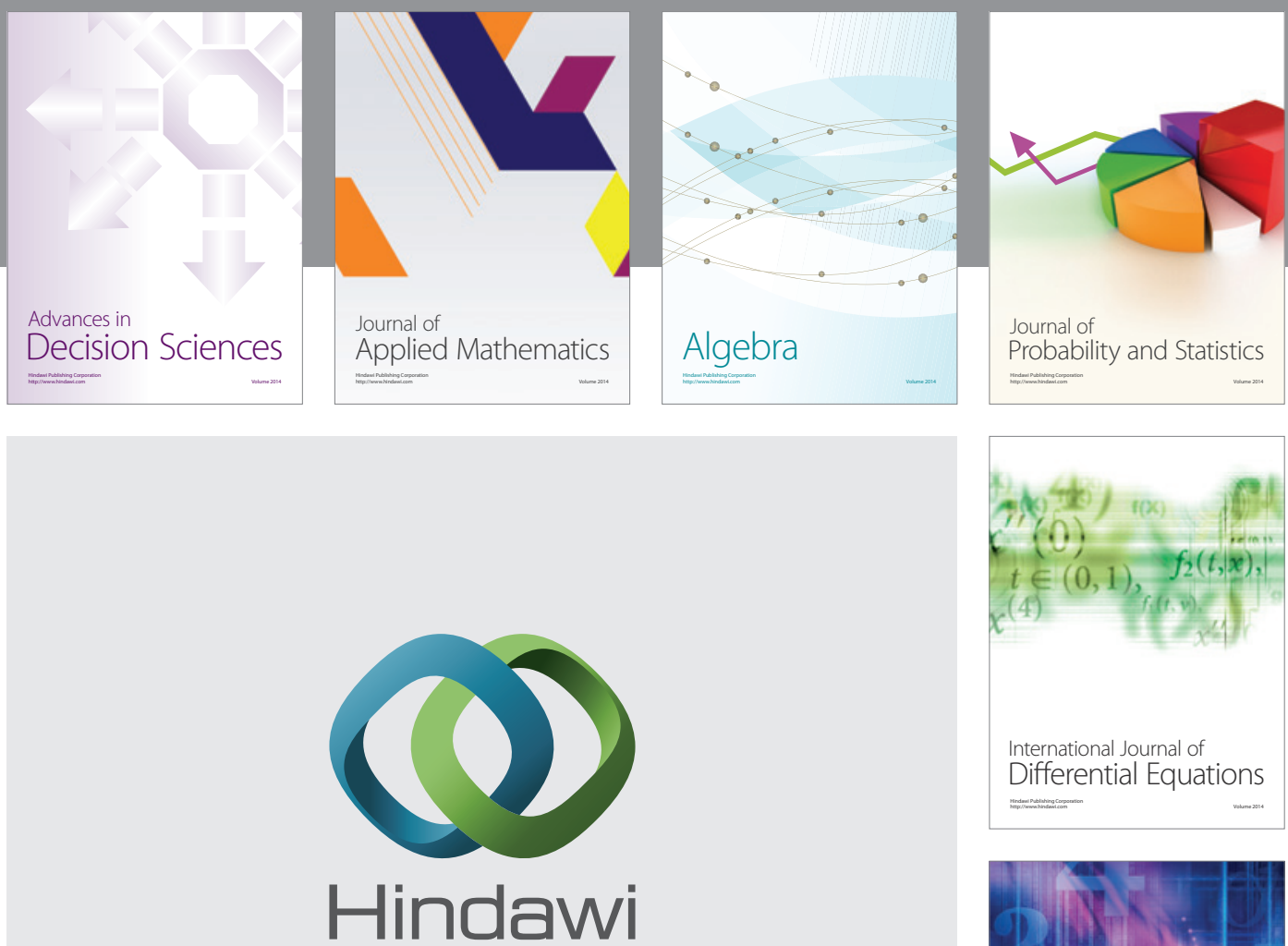

Submit your manuscripts at http://www.hindawi.com
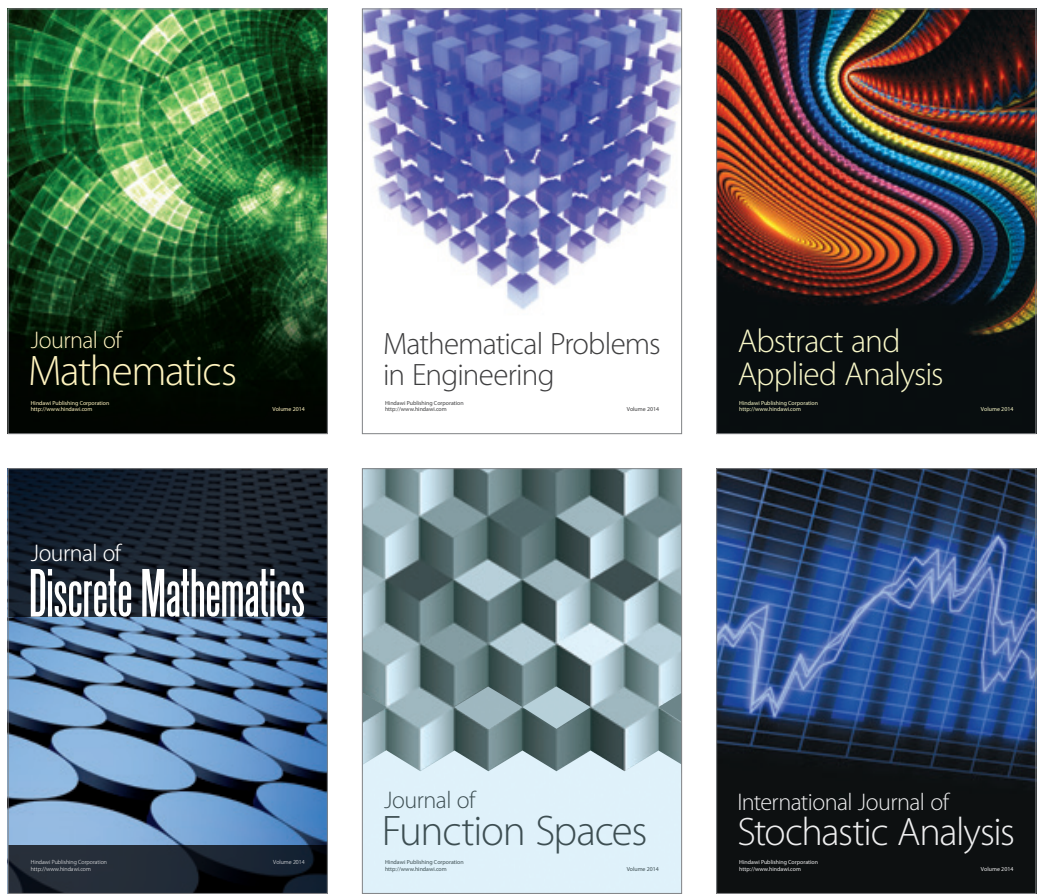

Journal of

Function Spaces

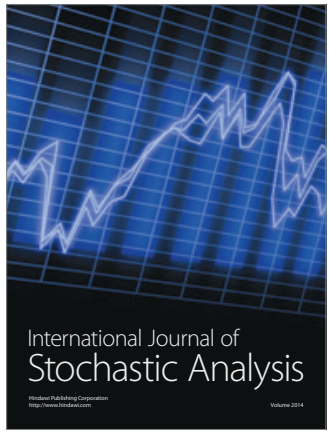

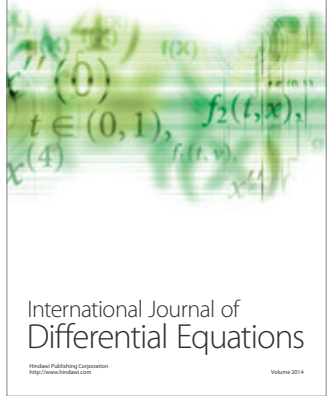
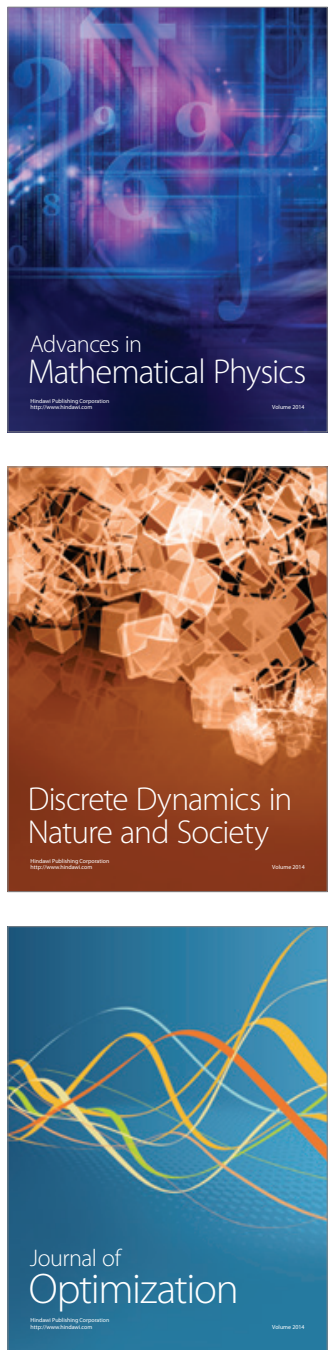